\title{
The Phenomenology of Iron Pnictides Superconductors Explained in the Framework of $s$-Wave Three-Band Eliashberg Theory
}

\author{
G. A. Ummarino \\ Dipartimento di Fisica and CNISM, Politecnico di Torino, Corso Duca degli Abruzzi 24, 10129 Torino, Italy
}

Correspondence should be addressed to G. A. Ummarino, giovanni.ummarino@infm.polito.it

Received 25 May 2009; Revised 10 August 2009; Accepted 3 September 2009

Academic Editor: Igor Mazin

Copyright ( $) 2010$ G. A. Ummarino. This is an open access article distributed under the Creative Commons Attribution License, which permits unrestricted use, distribution, and reproduction in any medium, provided the original work is properly cited.

\begin{abstract}
The $s$-wave three-band Eliashberg theory can simultaneously reproduce the experimental critical temperatures and the gap values of the superconducting materials $\mathrm{LaFeAsO}_{0.9} \mathrm{~F}_{0.1}, \mathrm{Ba}_{0.6} \mathrm{~K}_{0.4} \mathrm{Fe}_{2} \mathrm{As}_{2}$ and $\mathrm{SmFeAsO}_{0.8} \mathrm{~F}_{0.2}$ as exponent of the more important families of iron pnictides. In this model the dominant role is played by interband interactions and the order parameter undergoes a sign reversal between hole and electron bands ( $s \pm$-wave symmetry). The values of all the gaps (with the exact experimental critical temperature) can be obtained by using high values of the electron-boson coupling constants and small typical boson energies (in agreement with experiments).
\end{abstract}

The discovery of Fe-based pnictide superconductors [13] has aroused great interest in the scientific community. For the first time noncuprate superconductor shows high critical temperature. In these systems, as in cuprates, the superconductivity occurs upon charge doping of a magnetic parent compound above a certain critical value. The more relevant difference is that in cuprates the parent compound is a Mott insulator with localized charge carriers and a strong Coulomb repulsion between electrons, while in the pnictides it is a bad metal and shows a tetragonal to orthorhombic structural transition below $\approx 140 \mathrm{~K}$, followed by the onset of an antiferromagnetic spin-density-wave ordering [4]. Charge doping gives rise to superconductivity and, at the same time, inhibits the occurrence of both the static magnetic order and the structural transition. The Fermi surface consists of two or three hole-like sheets around the $\Gamma$ point in the first Brillouin zone and two electron-like sheets around $M$ point. Up to now, the most intensively studied systems are the 1111 compounds, $\mathrm{ReFeAsO}_{1-x} \mathrm{~F}_{x}(\mathrm{Re}=\mathrm{La}, \mathrm{Sm}, \mathrm{Nd}, \mathrm{Pr}$, etc. $)$ and the 122 ones, hole- or electron-doped $\mathrm{AFe}_{2} \mathrm{As}_{2}(\mathrm{~A}=\mathrm{Ba}, \mathrm{Sr}$, Ca).

At present it is not completely clear what is the microscopic pairing mechanism responsible for superconductivity.
The conventional phonon-mediated coupling mechanism is too week and cannot explain the observed high $T_{c}$ within the standard Migdal-Eliashberg theory $[5,6]$. The calculated $T_{c}$ increases only marginally with the inclusion of multiband effects and remains far from experimental values. On the other hand, the magnetic nature of the parent compound seems to favor a coupling mechanism based on nesting-related antiferromagnetic spin fluctuations [7]. In this case an interband sign reversal of the order parameter between different sheets of the Fermi surface ( $s \pm$ symmetry) is predicted. The number, amplitude, and symmetry of the superconducting energy gaps are indeed fundamental physical quantities that any microscopic model of superconductivity has to account for. Experiments with powerful techniques such as ARPES, point-contact spectroscopy, and STM, have been carried out to study the superconducting gaps in pnictides (for a review see [8]). Although the results are sometimes in disagreement with each other, a multigap scenario is emerging with evidence for rather high gap ratios, $\Delta_{1} / \Delta_{2} \approx 2-3$ [8]. A two-band BCS model cannot account either for the amplitude of the experimental gaps or for their ratio. Three-band BCS models have been investigated [911] which can reproduce the experimental gap ratio but not 


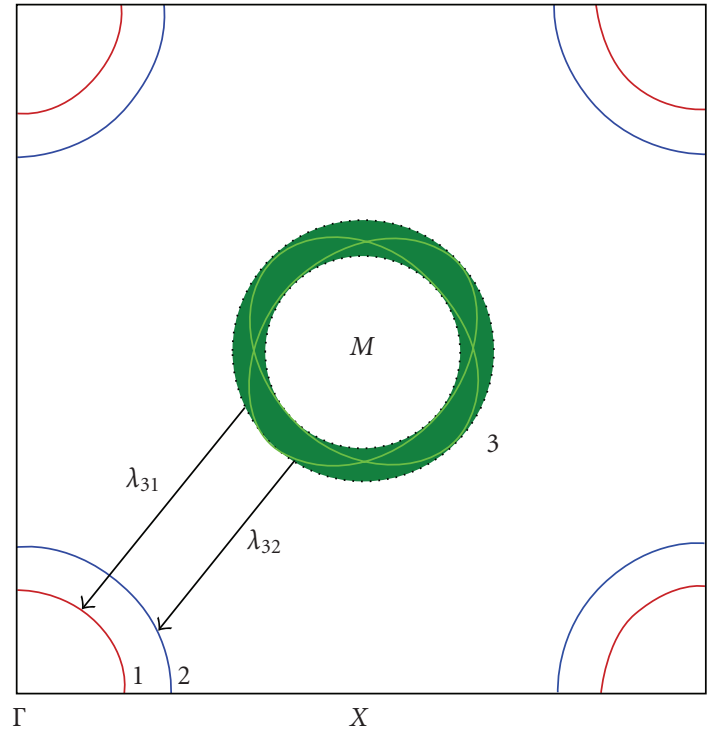

Figure 1: Schematic drawing of the multiband model used in this work. The two hole bands ( 1 and 2 ) are centered around the $\Gamma$ point, while the equivalent electron band (3) around the $M$ point of the reduced Brillouin zone.

the exact experimental gap values when the experimental $T_{c}$ is exactly reproduced. In this regard, a reliable study has to be carried out within the framework of the Eliashberg theory for strong coupling superconductors [12-18], due to the possible high values of the coupling constants necessary to explain the experimental data.

By using this strong-coupling approach, I show here that the superconducting iron pnictides represent a case of dominant negative interband-channel superconductivity ( $s \pm-$ wave symmetry) with high values of the electron-boson coupling constants and small typical boson energies. Furthermore I prove that a small contribution of intraband coupling does not significantly affect the obtained results and that the contribution of the Coulomb pseudopotential is negligible.

The electronic structure of pnictides can be approximately reproduced by using a three-band model (Figure 1) with two hole bands (1 and 2) and one equivalent electron band (3) [9]. The $s$-wave order parameters of the hole bands $\Delta_{1}$ and $\Delta_{2}$ have opposite sign with respect to that of the electron $\Delta_{3}$ [7]. In such systems intraband coupling could be provided by phonons while interband coupling by antiferromagnetic spin fluctuations which in a one-band system are always pair breaking but here, in a multiband system, the interband terms can contribute to increase the critical temperature. In the multiband Eliashberg equations the spin fluctuations term in the intraband channel has positive sign for the renormalization functions $Z_{i}$ and negative sign for the superconducting order parameters $\Delta_{i}$ thus leading to a strong reduction of $T_{c}$. However, if we consider negative interband contributions in the $\Delta_{i}$ equations, the final result can be an increase of the critical temperature [19].

The generalization of the Eliashberg theory [12-18] for multiband systems has already been used with success to study the $\mathrm{MgB}_{2}$ superconductor [20-23]. To obtain the gaps and the critical temperature within the $s$-wave, threeband Eliashberg equations, one has to solve six coupled equations for the gaps $\Delta_{i}\left(i \omega_{n}\right)$ and the renormalization functions $Z_{i}\left(i \omega_{n}\right)$, where $i$ is a band index that ranges between 1 and 3 (see Figure 1) and $\omega_{n}$ are the Matsubara frequencies. For completeness we included in the equations the nonmagnetic and magnetic impurity scattering rates in the Born approximation, $\Gamma_{i j}^{N}$ and $\Gamma_{i j}^{M}$. In the imaginary-axis formulation [24] the equations are

$$
\begin{aligned}
\omega_{n} Z_{i}\left(i \omega_{n}\right)= & \omega_{n}+\sum_{j}\left(\Gamma_{i j}^{N}+\Gamma_{i j}^{M}\right) N_{j}^{Z}\left(i \omega_{n}\right) \\
& +\pi T \sum_{m, j} \Lambda_{i j}^{Z}\left(i \omega_{n}, i \omega_{m}\right) N_{j}^{Z}\left(i \omega_{m}\right), \\
Z_{i}\left(i \omega_{n}\right) \Delta_{i}\left(i \omega_{n}\right)= & \sum_{j}\left(\Gamma_{i j}^{N}-\Gamma_{i j}^{M}\right) N_{j}^{\Delta}\left(i \omega_{n}\right) \\
& +\pi T \sum_{m, j}\left[\Lambda_{i j}^{\Delta}\left(i \omega_{n}, i \omega_{m}\right)-\mu_{i j}^{*}\left(\omega_{c}\right)\right] \\
& \times \theta\left(\omega_{c}-\left|\omega_{m}\right|\right) N_{j}^{\Delta}\left(i \omega_{m}\right),
\end{aligned}
$$

where $\Lambda_{i j}^{Z}\left(i \omega_{n}, i \omega_{m}\right)=\Lambda_{i j}^{\mathrm{ph}}\left(i \omega_{n}, i \omega_{m}\right)+\Lambda_{i j}^{\mathrm{sp}}\left(i \omega_{n}, i \omega_{m}\right)$, $\Lambda_{i j}^{\Delta}\left(i \omega_{n}, i \omega_{m}\right)=\Lambda_{i j}^{\mathrm{ph}}\left(i \omega_{n}, i \omega_{m}\right)-\Lambda_{i j}^{\mathrm{sp}}\left(i \omega_{n}, i \omega_{m}\right) . \theta$ is the Heaviside function and $\omega_{c}$ is a cut-off energy. In particular, $\Lambda_{i j}^{\mathrm{ph}, \mathrm{sp}}\left(i \omega_{n}, i \omega_{m}\right)=2 \int_{0}^{+\infty} d \Omega \Omega \alpha_{i j}^{2} F^{\mathrm{ph}, \mathrm{sp}}(\Omega) /\left[\left(\omega_{n}-\omega_{m}\right)^{2}+\Omega^{2}\right]$, where ph means "phonon" and sp "spin fluctuations." Finally, $N_{j}^{\Delta}\left(i \omega_{m}\right)=\Delta_{j}\left(i \omega_{m}\right) / \sqrt{\omega_{m}^{2}+\Delta_{j}^{2}\left(i \omega_{m}\right)}$ and $N_{j}^{Z}\left(i \omega_{m}\right)=$ $\omega_{m} / \sqrt{\omega_{m}^{2}+\Delta_{j}^{2}\left(i \omega_{m}\right)}$.

In the real axis formulation the multiband $s$-wave Eliashberg equations $[25,26]$ are

$$
\begin{aligned}
& \omega Z_{i}(\omega) \\
& =\omega+\sum_{j}\left(\Gamma_{i j}^{N}+\Gamma_{i j}^{M}\right) N_{j}^{Z}(\omega) \\
& \quad+\sum_{j} \int_{-\infty}^{+\infty} d \omega^{\prime} \Lambda_{i j}^{Z}\left(\omega, \omega^{\prime}\right) \operatorname{Real}\left[N_{j}^{Z}\left(\omega^{\prime}\right)\right] 002 \mathrm{C}
\end{aligned}
$$

$$
\begin{aligned}
& Z_{i}(\omega) \Delta_{i}(\omega) \\
& =\sum_{j}\left(\Gamma_{i j}^{N}-\Gamma_{i j}^{M}\right) N_{j}^{\Delta}(\omega) \\
& \quad+\sum_{j} \int_{-\infty}^{+\infty} d \omega^{\prime}\left[\Lambda_{i j}^{\Delta}\left(\omega, \omega^{\prime}\right)-\mu_{i j}^{*}\left(\omega_{c}\right) \theta\left(\omega_{c}-\left|\omega^{\prime}\right|\right)\right] \\
& \quad \times \operatorname{Real}\left[N_{j}^{\Delta}\left(\omega^{\prime}\right)\right],
\end{aligned}
$$

where now $\Lambda_{i j}^{Z}(\omega, \omega)=\Lambda_{i j}^{\mathrm{ph}}\left(\omega, \omega^{\prime}\right)+\Lambda_{i j}^{\mathrm{sp}}\left(\omega, \omega^{\prime}\right), \Lambda_{i j}^{\Delta}\left(\omega, \omega^{\prime}\right)=$ $\Lambda_{i j}^{\mathrm{ph}}\left(\omega, \omega^{\prime}\right)-\Lambda_{i j}^{\mathrm{sp}}\left(\omega, \omega^{\prime}\right)$. In particular, $\Lambda_{i j}^{\mathrm{ph}, \mathrm{sp}}\left(\omega, \omega^{\prime}\right)=$ $\int_{0}^{+\infty} d \Omega\left(\alpha_{i j}^{2} F^{\mathrm{ph}, \mathrm{sp}}(\Omega) / 2\right)\left[\left(\tanh \left(\omega^{\prime} / 2 T\right)+\operatorname{coth}(\Omega / 2 T)\right) /\left(\omega^{\prime}+\right.\right.$ $\left.\Omega-\omega-i \delta)-\left(\tanh \left(\omega^{\prime} / 2 T\right)-\operatorname{coth}(\Omega / 2 T)\right) /\left(\omega^{\prime}-\Omega-\omega-i \delta\right)\right]$, 
and $N_{j}^{\Delta}(\omega)=\Delta_{j}(\omega) / \sqrt{\omega^{2}-\Delta_{j}^{2}(\omega)}$ and $N_{j}^{Z}(\omega)=$ $\omega / \sqrt{\omega^{2}-\Delta_{j}^{2}(\omega)}$.

In principle the solution of the three-band Eliashberg equations shown in (1) (or (2)) requires a huge number of input parameters: (i) nine electron-phonon spectral functions, $\alpha_{i j}^{2} F^{\mathrm{ph}}(\Omega)$; (ii) nine electron-SF spectral functions, $\alpha_{i j}^{2} F^{\mathrm{sp}}(\Omega)$; (iii) nine elements of the Coulomb pseudopotential matrix, $\mu_{i j}^{*}\left(\omega_{c}\right)$; (iv) nine nonmagnetic $\Gamma_{i j}^{N}$ and nine paramagnetic $\Gamma_{i j}^{M}$ impurity scattering rates.

It is obvious that a practical solution of these equations requires a drastic reduction in the number of free parameters of the model. According to the work of Mazin et al. [7] I know that (i) $\lambda_{i i}^{\mathrm{ph}} \gg \lambda_{i j}^{\mathrm{ph}} \approx 0$, that is, phonons mainly provide intraband coupling but the total electron-phonon coupling constant $\sum_{i} \lambda_{i i}^{\mathrm{ph}}$ should be very small $[5,6]$, (ii) $\lambda_{i j}^{\mathrm{sp}} \gg \lambda_{i i}^{\mathrm{sp}} \approx$ 0 , that is, SF mainly provide interband coupling. I include these features in the simplest three-band model by posing $\lambda_{i i}^{\mathrm{ph}}=\lambda_{i j}^{\mathrm{ph}}=0, \lambda_{i i}^{\mathrm{sp}}=0$, and $\mu_{i i}^{*}\left(\omega_{c}\right)=\mu_{i j}^{*}\left(\omega_{c}\right)=0$. Here, of course, it is $\lambda_{i j}^{\mathrm{ph}, \mathrm{sp}}=2 \int_{0}^{+\infty} d \Omega\left(\alpha_{i j}^{2} F^{\mathrm{ph}, \mathrm{sp}}(\Omega) / \Omega\right)$. Moreover, I set $\Gamma_{i j}^{N}=\Gamma_{i j}^{M}=0$ in (1)-(2) and (3)-(4).

Within these approximations, the electron-boson coupling-constant matrix $\lambda_{i j}=\lambda_{i j}^{\text {sp }}$ becomes [9]

$$
\left(\begin{array}{ccc}
0 & 0 & \lambda_{13}=\lambda_{31} \nu_{1} \\
0 & 0 & \lambda_{23}=\lambda_{32} \nu_{2} \\
\lambda_{31} & \lambda_{32} & 0
\end{array}\right),
$$

where $v_{1}=N_{1}(0) / N_{3}(0), v_{2}=N_{2}(0) / N_{3}(0)$, and $N_{i}(0)$ is the normal density of states at the Fermi level for the $i$-band $(i=$ 1, 2, 3 according to Figure 1 ).

I initially solved the Eliashberg equations on the imaginary axis to calculate the critical temperature and, by means of the technique of the Padè approximants $[27,28]$, to obtain the low-temperature value of the gaps because in presence of a strong coupling interaction or of impurities, the value of $\Delta_{i}\left(i \omega_{n=0}\right)$ obtained by solving the imaginary-axis Eliashberg equations can be very different from the value of $\Delta_{i}$ obtained from the real-axis Eliashberg equations [29]. I also solved the three-band Eliashberg equations in the real-axis formulation.

I reproduced the critical temperature and the gap values in three representative cases: (i) the compound $\mathrm{LaFeAsO}_{0.9} \mathrm{~F}_{0.1}$ with $\mathrm{T}_{c}=27 \mathrm{~K}$ where point-contact spectroscopy measurements gave $\Delta_{1}(0) \approx 3.8 \mathrm{meV}$ and $\Delta_{2}(0) \approx$ $8.0 \mathrm{meV}$ [30]; (ii) the compound $\mathrm{Ba}_{0.6} \mathrm{~K}_{0.4} \mathrm{Fe}_{2} \mathrm{As}_{2}$ with $T_{c}=$ $37 \mathrm{~K}$ where ARPES measurements gave $\Delta_{1}(0) \approx 12.1 \mathrm{meV}$, $\Delta_{2}(0) \approx 5.5 \mathrm{meV}$, and $\Delta_{3}(0) \approx 12.8 \mathrm{meV}$ [31]; (iii) the compound $\mathrm{SmFeAsO}_{0.8} \mathrm{~F}_{0.2}$ with $\mathrm{T}_{c}=52 \mathrm{~K}$ where, according to point-contact spectroscopy measurements, $\Delta_{1}(0) \approx$ $17.0 \mathrm{meV}$ and $\Delta_{2}(0) \approx 5.7 \mathrm{meV}[32,33]$.

Inelastic neutron-scattering experiments suggest that the typical boson energy possibly responsible for superconductivity ranges roughly between 10 and $30 \mathrm{meV}[34,35]$. In the numerical simulations I used spectral functions with Lorentzian shape, that is, $\alpha_{i j}^{2} F(\Omega)=C_{i j}\left[L\left(\Omega+\Omega_{i j}, Y_{i j}\right)-\right.$ $\left.L\left(\Omega-\Omega_{i j}, Y_{i j}\right)\right]$, where $L\left(\Omega \pm \Omega_{i j}, Y_{i j}\right)=\left[\left(\Omega \pm \Omega_{i j}\right)^{2}+\right.$ $\left.\left(Y_{i j}\right)^{2}\right]^{-1}, C_{i j}$ are the normalization constants necessary to

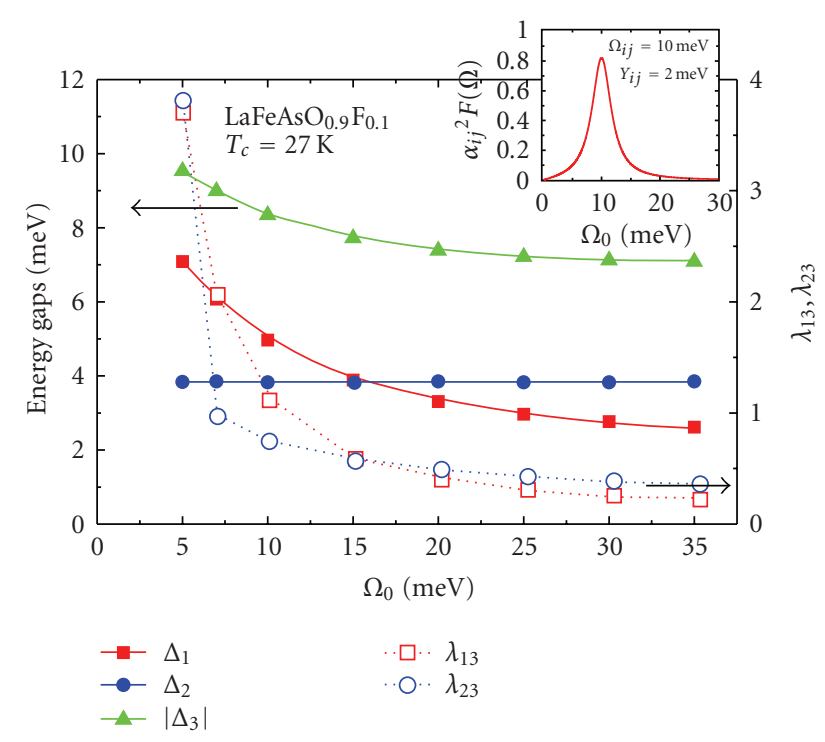

FIGURE 2: Full symbols, left axis: calculated gap values at $T=2 \mathrm{~K}$ for $\mathrm{LaFeAsO}_{0.9} \mathrm{~F}_{0.1}$ as a function of typical boson energy $\Omega_{0}$. Open symbols, right axis: electron-boson coupling constants, $\lambda_{13}$ and $\lambda_{23}$, as a function of $\Omega_{0}$. The inset shows the spectral function used in this model in the case $\Omega_{i j}=10 \mathrm{meV}$.

obtain the proper values of $\lambda_{i j}$ while $\Omega_{i j}$ and $Y_{i j}$ are the peak energies and half-widths, respectively. In all the calculations I always set $\Omega_{i j}=\Omega_{0}$, with $\Omega_{0}$ ranging between 5 and $35 \mathrm{meV}$ and $Y_{i j}=2 \mathrm{meV}$. The cut-off energy is $\omega_{c}=12 \cdot \Omega_{0}$ and the maximum quasiparticle energy is $\omega_{\max }=16 \cdot \Omega_{0}$.

In the $T_{c}=27 \mathrm{~K}$ case I know that $\nu_{1}=0.03$ and $\nu_{2}=1$ [36] while in the $T_{c}=37 \mathrm{~K}$ case $\nu_{1}=1$ and $\nu_{2}=2$ [9] and in the $T_{c}=52 \mathrm{~K}$ case I have $v_{1}=0.4$ and $v_{2}=0.5$ [37]. Once the energy of the boson peak $\Omega_{0}$ is set, only two free parameters are left in the model: $\lambda_{31}$ and $\lambda_{32}$.

By properly selecting the values of these parameters it is relatively easy to obtain the experimental values of the critical temperature and of the small gap, which is well known. It is more difficult to reproduce the values of the large gaps of bands 1 and 3 since, due to the high $2 \Delta_{1,3} / k_{B} T_{c}$ ratio (of the order of 8-9), high values of the coupling constants and small boson energies are required. Figures 2, 3, and 4 show the values of the calculated gaps (full symbols, left axis) as a function of the boson peak energy, $\Omega_{0}$. The corresponding values of $\lambda_{13}$ and $\lambda_{12}$, chosen in order to reproduce the values of $T_{c}$ and of the small gap, $\Delta_{2}$, are also shown in the figure (open symbols, right axis). In all materials examined, only when $\Omega_{0} \leq 10 \mathrm{meV}$ the values of the large gap correspond to the experimental data. Indeed, when $\Omega_{0}$ increases, the values of $\Delta_{1}$ and $\Delta_{3}$ strongly decrease. As a consequence, a rather small energy of the boson peak together with a very strong coupling (particularly in the 3-1 channel) is needed in order to obtain the experimental $T_{c}$ and the correct gap values. In this regard, it is worth noticing that the absolute values of the large gaps cannot be reproduced in a interband-only, twoband Eliashberg model [38], as well as within a three-band BCS model. In the latter case it is only possible to obtain a ratio of the gaps close to the experimental one $[10,11]$. 
TABLE 1: The effect of the intraband terms and Coulomb interaction on the gap values.

\begin{tabular}{lccc}
\hline Pure interband & $\lambda_{i i}=0$. & $\mu_{i j}^{*}=0$ & $\Omega_{0}=10 \mathrm{meV}$ \\
Free parameters & $\lambda_{31}=4.267$ & $\lambda_{32}=1.138$ & - \\
Results & $\Delta_{1}=10.29 \mathrm{meV}$ & $\Delta_{2}=5.56 \mathrm{meV}$ & $\Delta_{3} \mid=10.22 \mathrm{meV}$ \\
Intraband & $\lambda_{i i}=0.4$ & $\mu_{i j}^{*}=0$ & $\Omega_{0}=10 \mathrm{meV}$ \\
Free parameters & $\lambda_{31}=3.866$ & $\lambda_{32}=0.471$ & - \\
Results & $\Delta_{1}=10.30 \mathrm{meV}$ & $\Delta_{2}=5.62 \mathrm{meV}$ & $\left|\Delta_{3}\right|=10.24 \mathrm{meV}$ \\
Intraband and Coulomb & $\lambda_{i i}=0.4$ & $\mu_{i j}^{*}=0.1$ & $\Omega_{0}=10 \mathrm{meV}$ \\
Free parameters & $\lambda_{31}=2.730$ & $\lambda_{32}=0.758$ & - \\
Results & $\Delta_{1}=7.49 \mathrm{meV}$ & $\Delta_{2}=5.72 \mathrm{meV}$ & $\left|\Delta_{3}\right|=7.98 \mathrm{meV}$ \\
\hline
\end{tabular}

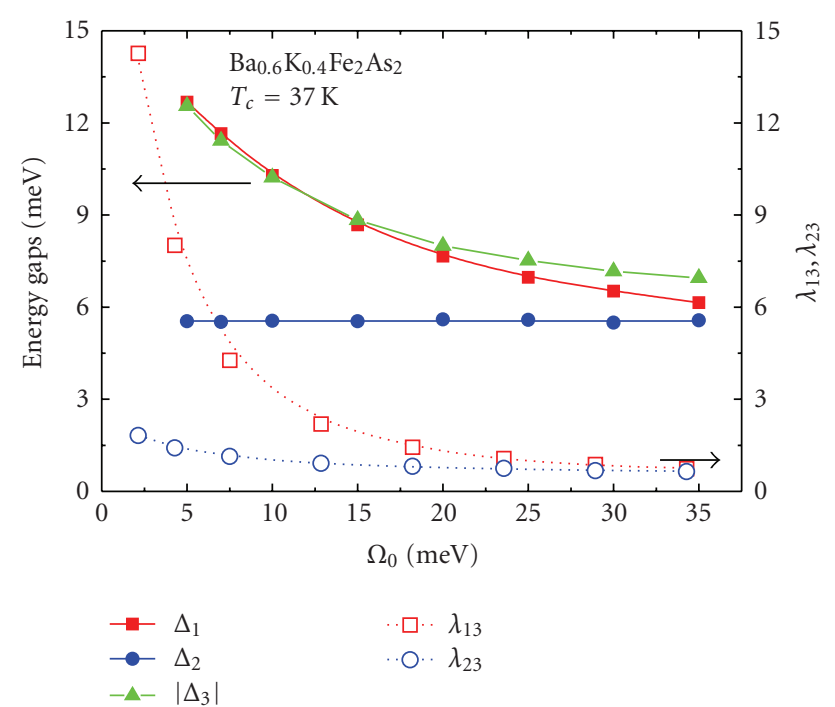

FIgURe 3: Full symbols, left axis: calculated gap values at $T=2 \mathrm{~K}$ for $\mathrm{Ba}_{0.6} \mathrm{~K}_{0.4} \mathrm{Fe}_{2} \mathrm{As}_{2}$ as a function of typical boson energy $\Omega_{0}$. Open symbols, right axis: electron-boson coupling constants, $\lambda_{13}$ and $\lambda_{23}$, as a function of $\Omega_{0}$.

I also tested the effect into the model of a small intraband coupling (possibly of phonon origin). In the case of $\mathrm{Ba}_{0.6} \mathrm{~K}_{0.4} \mathrm{Fe}_{2} \mathrm{As}_{2}\left(T_{c}=37 \mathrm{~K}\right)$ I fixed $\Omega_{0}=10 \mathrm{meV}$ and $\lambda_{i i}=0.4$ since I know indeed that this coupling cannot be very high $[5,6]$. Then I determined the free parameters $\lambda_{31}$ and $\lambda_{32}$ in order to obtain $T_{c}=37 \mathrm{~K}$. It might be thought that this term can sensibly contribute to increase the gap values but, as can be seen in Table 1, this is not the case as the gap values only show a slight increase.

The effect of Coulomb interaction was also investigated for the case shown in Table 1 where a weak intraband coupling is included. I chose $\mu_{i j}^{*}=\mu_{i i}^{*}=0.1$ and, as expected, I found that the intraband Coulomb pseudopotential has a negligible effect while the interband one [19] strongly contributes to raise $T_{c}$ and reduces in a considerable way [24] the value of $\lambda_{31}$. In this case, as shown in Table 1, it is only possible to obtain the correct value of the small gap because the electron-boson coupling is now too small and it is impossible to reproduce the value of the big gap. As a

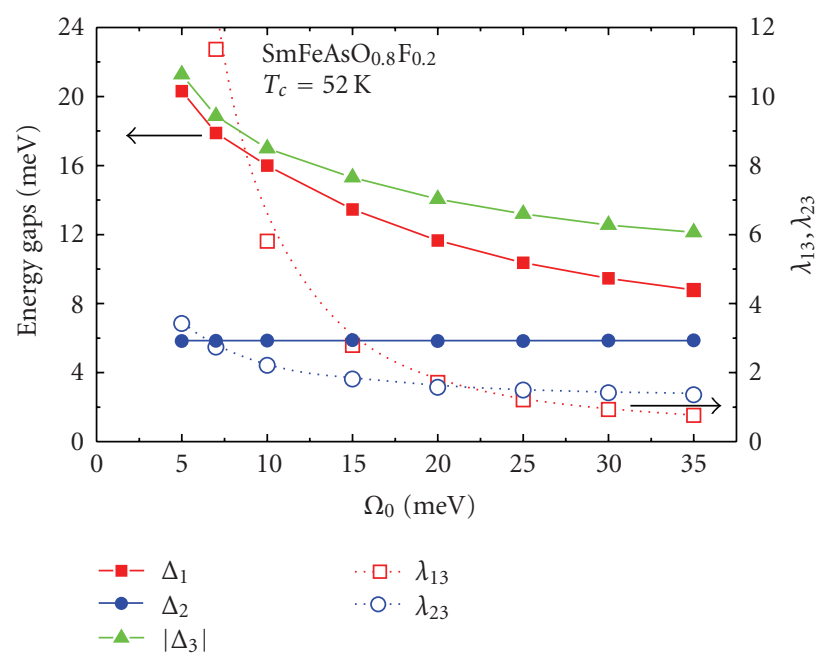

FIGURE 4: Full symbols, left axis: calculated gap values at $T=2 \mathrm{~K}$ for $\mathrm{SmFeAsO}_{0.8} \mathrm{~F}_{0.2}$ as a function of the typical boson energy $\Omega_{0}$. Open symbols, right axis: electron-boson coupling constants, $\lambda_{13}$ and $\lambda_{23}$, as a function of $\Omega_{0}$.

consequence, this result seems to exclude a strong interband Coulomb interaction in these compounds.

I also calculated the superconductive density of states in all three cases. The parameters used for the $T_{c}=27.00 \pm 0.01$ $\mathrm{K}$ case are $\Omega_{0}=10 \mathrm{meV}, \lambda_{13}=1.115$, and $\lambda_{32}=0.743$, for the $T_{c}=37.00 \pm 0.01 \mathrm{~K}$ case $\Omega_{0}=10 \mathrm{mev}, \lambda_{13}=4.267$, and $\lambda_{23}=1.138$ and for $T_{c}=52.00 \pm 0.01 \mathrm{~K}$ case $\Omega_{0}=10 \mathrm{mev}$, $\lambda_{13}=5.808$, and $\lambda_{23}=2.208$.

The value of coupling constant $\lambda_{31}$ is in the range 1-6 and this fact, at a first glance, may seem very unusual but these systems have some peculiarities in common with the heavy fermions superconductors. For example, in the compound $\mathrm{LaFeAsO}_{0.9} \mathrm{~F}_{0.1}$, the normal state at $T_{c}$ is asymmetric and pseudogapped, with two broad maxima that are progressively smoothed out on increasing the temperature [30]. This shape is very similar to that observed by point contact spectroscopy in materials with long-range spin-density-wave order, like $\mathrm{URu}_{2} \mathrm{Si}_{2}[39,40]$. The calculated superconductive normalized conductances are shown in Figure 5; the presence 


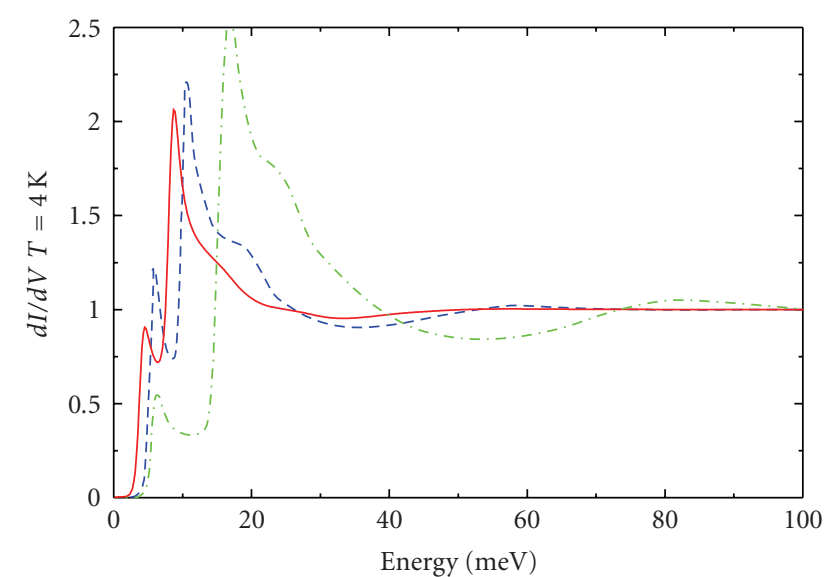

Figure 5: Calculated energy dependence of the superconductive normalized tunneling conductance at $T=4 \mathrm{~K}$ for $\mathrm{LaFeAsO}_{0.9} \mathrm{~F}_{0.1}$ ( $T_{c}=27 \mathrm{~K}$, red solid line), $\mathrm{Ba}_{0.6} \mathrm{~K}_{0.4} \mathrm{Fe}_{2} \mathrm{As}_{2}\left(T_{c}=37 \mathrm{~K}\right.$, dash blue line), and for $\mathrm{SmFeAsO}_{0.8} \mathrm{~F}_{0.2}\left(T_{c}=52 \mathrm{~K}\right.$, green dash-dot line $)$.

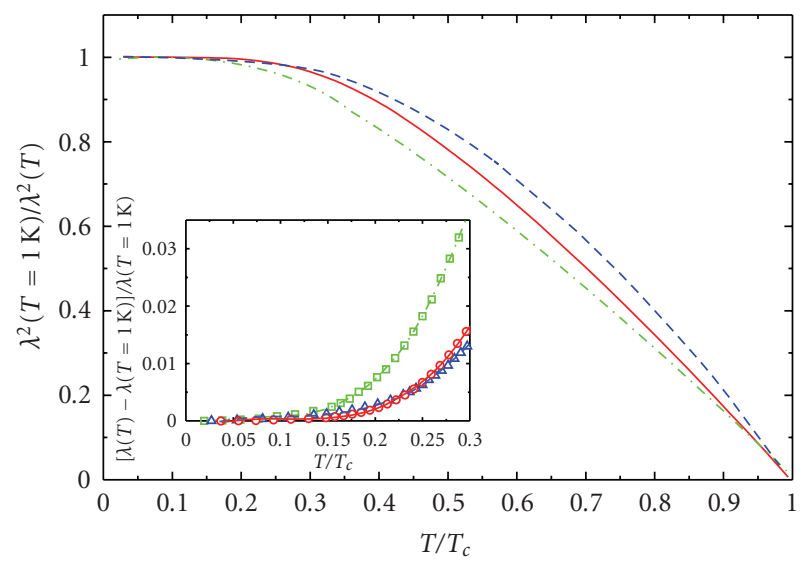

Figure 6: Calculated temperature dependence of the penetration depth $\left([\lambda(T) / \lambda(T=1 \mathrm{~K})]^{2}\right)$ for $\mathrm{LaFeAsO}_{0.9} \mathrm{~F}_{0.1}\left(T_{c}=27 \mathrm{~K}\right.$, red solid line), $\mathrm{Ba}_{0.6} \mathrm{~K}_{0.4} \mathrm{Fe}_{2} \mathrm{As}_{2}\left(T_{c}=37 \mathrm{~K}\right.$, dash blue line), and for $\mathrm{SmFeAsO}_{0.8} \mathrm{~F}_{0.2}\left(T_{c}=52 \mathrm{~K}\right.$, green dash-dot line $)$. The insert shows the behaviour of $[\lambda(T)-\lambda(T=1 \mathrm{~K})] / \lambda(T=1 \mathrm{~K})$ obtained by solution of Eliashberg equations: red circles for $\mathrm{LaFeAsO}_{0.9} \mathrm{~F}_{0.1}$, dark blue triangle for $\mathrm{Ba}_{0.6} \mathrm{~K}_{0.4} \mathrm{Fe}_{2} \mathrm{As}_{2}$, green squares for $\mathrm{SmFeAs} \mathrm{O}_{0.8} \mathrm{~F}_{0.2}$. The lines are the third-order polynomial fits.

of a hump at $\Omega \approx \Omega_{0}+\Delta_{1}$ is a typical strong coupling effect [41]. This feature, of course, is more evident when $\lambda_{13}$ is bigger. By cause of thermal broadening it is impossible to separate the peaks of the gaps $\Delta_{1}$ and $\Delta_{3}$.

The penetration depth as a function of temperature has been calculated in the three cases and is reported in Figure 6. It is in qualitative agree with the experimental data [42]. The inset shows the behaviour of $[\lambda(T)-\lambda(T=1 \mathrm{~K})] / \lambda(T=1 \mathrm{~K})$ at low temperature. Although at sufficiently low temperature an exponential fit may be certainly possible the inset shows that, on a larger $T$ range (up to $T / T_{c} \sim 0.3$ ) these curves can be best fitted by a third-order polynomial as experimentally observed [42].
In conclusion, I have shown that the newly discovered iron pnictides very likely represent a case of dominant negative interband-channel pairing superconductivity where an electron-boson coupling, such as the electron-spin fluctuactions one, can become a fundamental ingredient to increase $T_{c}$ in a multiband strong-coupling picture. In particular, the present results prove that a simple threeband model in strong-coupling regime can reproduce in a quantitative way the experimental $T_{c}$ and the energy gaps of the pnictide superconductors with only two free parameters, $\lambda_{31}$ and $\lambda_{32}$, provided that the typical energies of the spectral functions are of the order of $10 \mathrm{meV}$ and the coupling constants are very high $\left(1<\lambda_{31}<6\right)$.

\section{Acknowledgments}

The author thanks I. I. Mazin for useful discussions, Mauro Tortello and Dario Daghero for manuscript revision.

\section{References}

[1] Y. Kamihara, T. Watanabe, M. Hirano, and H. Hosono, "Ironbased layered superconductor $\mathrm{La}\left[\mathrm{O}_{1-x} \mathrm{~F}_{x}\right] \mathrm{FeAs}(x=0.05-$ 0.12) with $T_{c}=26 \mathrm{~K}$," Journal of the American Chemical Society, vol. 130, no. 11, pp. 3296-3297, 2008.

[2] Z.-A. Ren, W. Lu, J. Yang, et al., "Superconductivity at $55 \mathrm{~K}$ in iron-based F-doped layered quaternary compound Sm $\left[\mathrm{O}_{1-x} \mathrm{~F}_{x}\right]$ FeAs," Chinese Physics Letters, vol. 25, no. 6, pp. 2215-2216, 2008.

[3] M. Rotter, M. Tegel, and D. Johrendt, "Superconductivity at $38 \mathrm{~K}$ in the iron arsenide $\left(\mathrm{Ba}_{1-x} \mathrm{~K}_{x}\right) \mathrm{Fe}_{2} \mathrm{As}_{2}$," Physical Review Letters, vol. 101, no. 10, Article ID 107006, 2008.

[4] C. de La Cruz, Q. Huang, J. W. Lynn, et al., "Magnetic order close to superconductivity in the iron-based layered $\mathrm{LaO}_{1-x} \mathrm{~F}_{x} \mathrm{FeAs}$ systems," Nature, vol. 453, no. 7197, pp. 899902, 2008 .

[5] L. Boeri, O. V. Dolgov, and A. A. Golubov, "Is $\mathrm{LaFeAsO}_{1-x} \mathrm{~F}_{x}$ an electron-phonon superconductor?" Physical Review Letters, vol. 101, no. 2, Article ID 026403, 4 pages, 2008.

[6] L. Boeri, O. V. Dolgov, and A. A. Golubov, "Electron-phonon properties of pnictide superconductors," Physica C, vol. 469, no. 9-12, pp. 628-634, 2009.

[7] I. I. Mazin, D. J. Singh, M. D. Johannes, and M. H. Du, "Unconventional superconductivity with a sign reversal in the order parameter of $\mathrm{LaFeAsO}_{1-x} \mathrm{~F}_{x}$ " Physical Review Letters, vol. 101, no. 5, Article ID 057003, 2008.

[8] Physica C 469, 2009, Special Issue on Pnictides.

[9] I. I. Mazin and J. Schmalian, "Pairing symmetry and pairing state in ferropnictides: theoretical overview," Physica C, vol. 469, no. 9-12, pp. 614-627, 2009.

[10] L. Benfatto, M. Capone, S. Caprara, C. Castellani, and C. di Castro, "Multiple gaps and superfluid density from interband pairing in a four-band model of the iron oxypnictides," Physical Review B, vol. 78, no. 14, Article ID 140502, 4 pages, 2008.

[11] E. Z. Kuchinskii and M. V. Sadovskii, "Multiple bands: a key to high-temperature superconductivity in iron arsenides?" Journal of Experimental and Theoretical Physics Letters, vol. 11, no. 3, pp. 156-160, 2009.

[12] G. M. Eliashberg, "Interactions between electrons and lattice vibrations in a superconductor," Soviet Physycs. JETP Letters, vol. 11, no. 3, pp. 696-702, 1960. 
[13] D. J. Scalapino, "The Electron-phonon interaction and strongcoupling superconductors," in Superconductivity, R. D. Parks, Ed., p. 449, Marcel Dekker, New York, NY, USA, 1969.

[14] J. P. Carbotte, "Properties of boson-exchange superconductors," Reviews of Modern Physics, vol. 62, no. 4, pp. 1027-1157, 1990.

[15] P. B. Allen and B. Mitrovich, "Theory of superconducting $T_{c}$ ", in Solid State Physics, H. Ehrenreich, F. Seitz, and D. Turnbull, Eds., vol. 37, Academic Press, New York, NY, USA, 1982.

[16] F. Marsiglio and J. P. Carbotte, "Electron-phonon superconductivity," in The Physics of Conventional and Unconventional Superconductors, K. H. Bennemann and J. B. Ketterson, Eds., p. 233, Springer, New York, NY, USA, 2003.

[17] F. Marsiglio, "Eliashberg theory of the critical temperature and isotope effect. Dependence on bandwidth, band-filling, and direct Coulomb repulsion," Journal of Low Temperature Physics, vol. 87, no. 5-6, pp. 659-682, 1992.

[18] C. R. Leavens and E. Talbot, "Effect of thermal phonons on the superconducting transition temperature," Physical Review B, vol. 28, no. 3, pp. 1304-1313, 1983.

[19] G. A. Ummarino, "Effects of magnetic impurities on twoband superconductor," Journal of Superconductivity and Novel Magnetism, vol. 20, no. 7-8, pp. 639-642, 2007.

[20] S. V. Shulga, S.-L. Drechsler, G. Fuchs, et al., "Upper critical field peculiarities of superconducting $\mathrm{YnI}_{2} \mathrm{~B}_{2} \mathrm{C}$ and $\mathrm{LuNi}_{2} \mathrm{~B}_{2}$ C," Physical Review Letters, vol. 80, no. 8, pp. 1730$1733,1998$.

[21] S. D. Adrian, S. A. Wolf, O. V. Dolgov, S. Shulga, and V. Z. Kresin, "Density of states and the energy gap in superconducting cuprates," Physical Review B, vol. 56, no. 13, pp. 7878-7881, 1997.

[22] G. A. Ummarino, R. S. Gonnelli, S. Massidda, and A. Bianconi, "Two-band Eliashberg equations and the experimental $T_{c}$ of the diboride $\mathrm{Mg}_{1-x} \mathrm{AI}_{x} \mathrm{~B}_{2}$, " Physica $C$, vol. 407, no. 3-4, pp. 121-127, 2004.

[23] E. J. Nicol and J. P. Carbotte, "Properties of the superconducting state in a two-band model," Physical Review B, vol. 71, no. 5, Article ID 054501, 18 pages, 2005.

[24] G. A. Ummarino, "Iron-based layered compounds: the effect of negative interband coupling," Journal of Superconductivity and Novel Magnetism, vol. 22, no. 6, pp. 603-607, 2009.

[25] O. V. Dolgov, R. K. Kremer, J. Kortus, A. A. Golubov, and S. V. Shulga, "Thermodynamics of two-band superconductors: the case of $\mathrm{MgB}_{2}$," Physical Review B, vol. 72, no. 2, Article ID 024504, 2005.

[26] D. Parker, O. V. Dolgov, M. M. Korshunov, A. A. Golubov, and I. I. Mazin, "Extended $s \pm$ scenario for the nuclear spin-lattice relaxation rate in superconducting pnictides," Physical Review $B$, vol. 78, no. 13, Article ID 134524, 5 pages, 2008.

[27] H. J. Vidberg and J. W. Serene, "Solving the Eliashberg equations by means of N-point Padé approximants," Journal of Low Temperature Physics, vol. 29, no. 3-4, pp. 179-192, 1977.

[28] C. R. Leavens and D. S. Ritchie, "Extension of the N-point Padé approximants solution of the Eliashberg equations to $\mathrm{T} \sim \mathrm{T}_{\mathrm{C}}$," Solid State Communications, vol. 53, no. 2, pp. 137142, 1985.

[29] G. A. Ummarino and R. S. Gonnelli, "Real-axis direct solution of the d-wave Eliashberg equations and the tunneling density of states in optimally doped $\mathrm{Bi}_{2} \mathrm{Sr}_{2} \mathrm{CaCu}_{2} \mathrm{O}_{8+x}$," Physica $C$, vol. 328, no. 3, pp. 189-194, 1999.

[30] R. S. Gonnelli, D. Daghero, M. Tortello, et al., "Coexistence of two order parameters and a pseudogaplike feature in the ironbased superconductor $\mathrm{LaFeAsO}_{1-x} \mathrm{~F}_{x}$," Physical Review B, vol. 79, no. 18, Article ID 184526, 11 pages, 2009.
[31] H. Ding, P. Richard, K. Nakayama, et al., "Observation of Fermi-surface-dependent nodeless superconducting gaps in $\mathrm{Ba}_{0.6} \mathrm{~K}_{0.4} \mathrm{Fe}_{2} \mathrm{As}_{2}$," Europhysics Letters, vol. 83, no. 4, Article ID 47001, 2008.

[32] D. Daghero, M. Tortello, R. S. Gonnelli, V. A. Stepanov, N. D. Zhigadlo, and J. Karpinski, "Evidence for two-gap nodeless superconductivity in $\mathrm{SmFeAsO}_{1-x} \mathrm{~F}_{x}$ from pointcontact Andreev-reflection spectroscopy," Physical Review B, vol. 80, no. 6, 2009.

[33] D. Daghero, M. Tortello, R. S. Gonnelli, et al., "Point-contact Andreev-reflection spectroscopy in $\mathrm{ReFeAsO}_{1-x} \mathrm{~F}_{x}(\mathrm{Re}=$ La,Sm): possible evidence for two nodeless gaps," Physica $C$, vol. 469, no. 9-12, pp. 512-520, 2009.

[34] A. D. Christianson, E. A. Goremychkin, R. Osborn, et al., "Unconventional superconductivity in $\mathrm{Ba}_{0.6} \mathrm{~K}_{0.4} \mathrm{Fe}_{2} \mathrm{As}_{2}$ from inelastic neutron scattering," Nature, vol. 456, no. 7224, pp. 930-932, 2008.

[35] R. Osborn, S. Rosenkranz, E. A. Goremychkin, and A. D. Christianson, "Inelastic neutron scattering studies of the spin and lattice dynamics in iron arsenide compounds," Physica $C$, vol. 469, no. 9-12, pp. 498-506, 2009.

[36] M. V. Sadovskii, "High-temperature superconductivity in iron-based layered iron compounds," Physics-Uspekhi, vol. 51, no. 12, pp. 1201-1227, 2008.

[37] I. I. Mazin, private communication.

[38] O. V. Dolgov, I. I. Mazin, D. Parker, and A. A. Golubov, "Interband superconductivity: contrasts between BardeenCooper-Schrieffer and Eliashberg theories," Physical Review B, vol. 79, no. 6, Article ID 060502, 2009.

[39] K. Hasselbach, J. R. Kirtley, and P. Lejay, "Point-contact spectroscopy of superconducting $\mathrm{URu}_{2} \mathrm{Si}_{2}$," Physical Review $B$, vol. 46, no. 9, pp. 5826-5829, 1992.

[40] R. Escudero, F. Morales, and P. Lejay, "Temperature dependence of the antiferromagnetic state in $\mathrm{URu}_{2} \mathrm{Si}_{2}$ by pointcontact spectroscopy," Physical Review B, vol. 49, no. 21, pp. 15271-15275, 1994.

[41] G. A. Ummarino, R. S. Gonnelli, and D. Daghero, "Tunneling conductance of SIN junctions with different gap symmetries and non-magnetic impurities by direct solution of real-axis Eliashberg equations," Physica C, vol. 377, no. 3, pp. 292-303, 2002.

[42] R. Prozorov, M. A. Tanatar, R. T. Gordon, et al., "Anisotropic London penetration depth and superfluid density in single crystals of iron-based pnictide superconductors," Physica $C$, vol. 469, no. 9-12, pp. 582-589, 2009. 

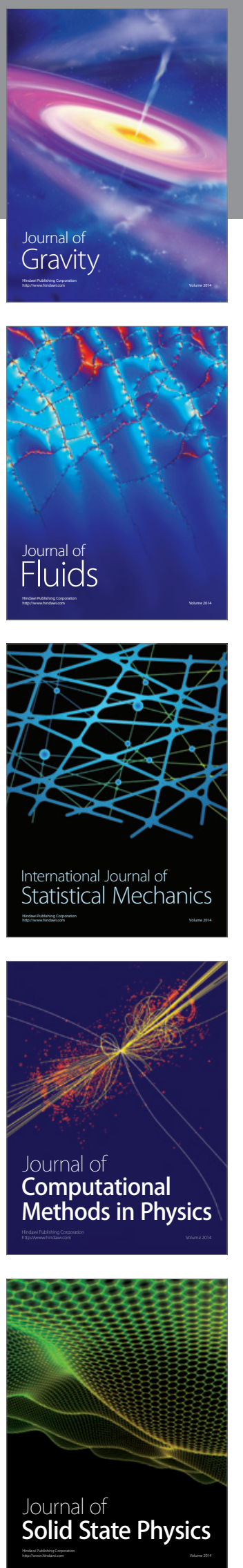

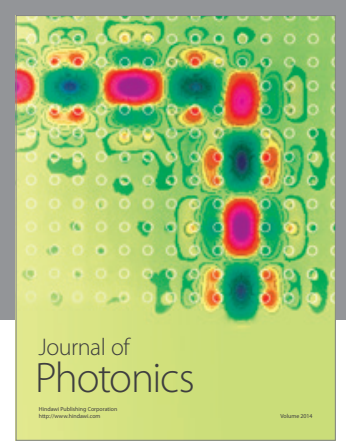

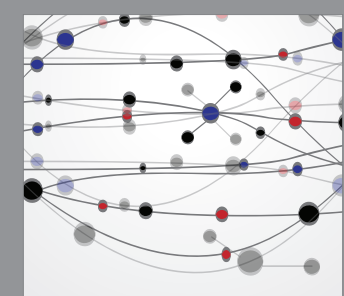

The Scientific World Journal
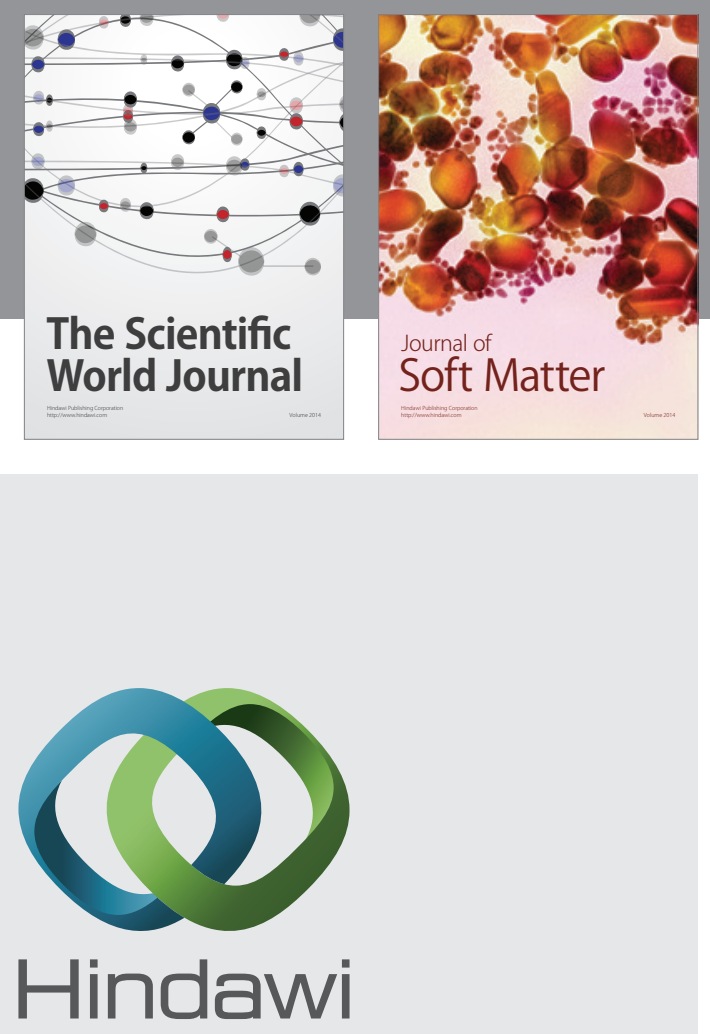

Submit your manuscripts at

http://www.hindawi.com
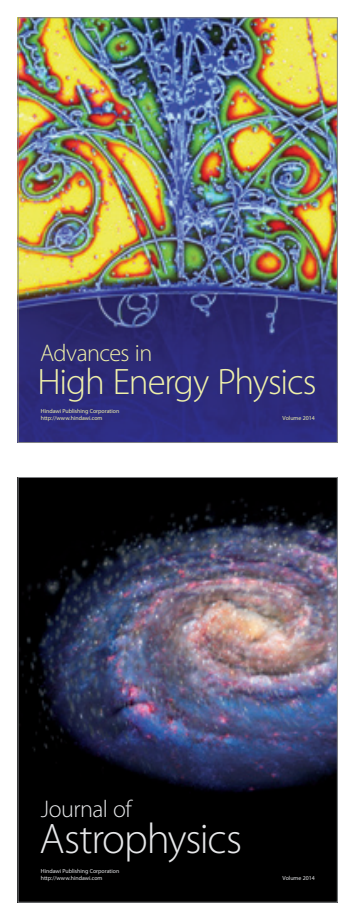
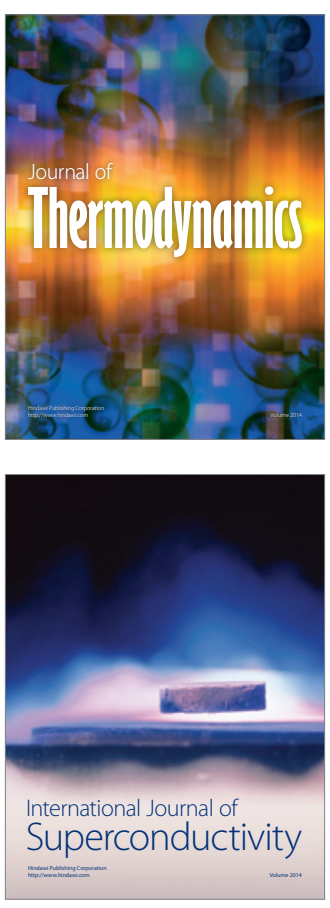
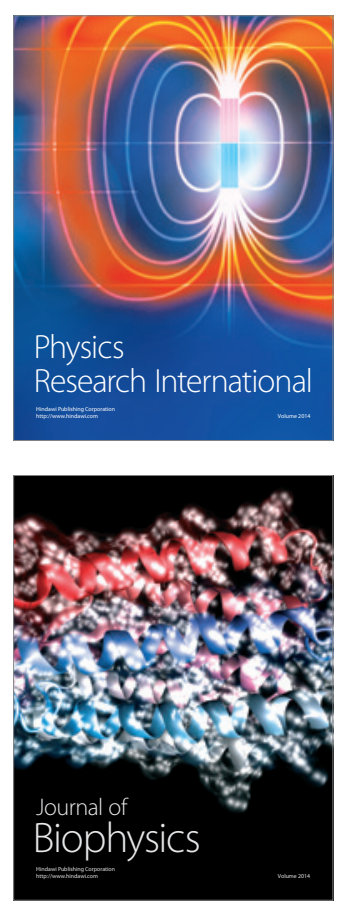
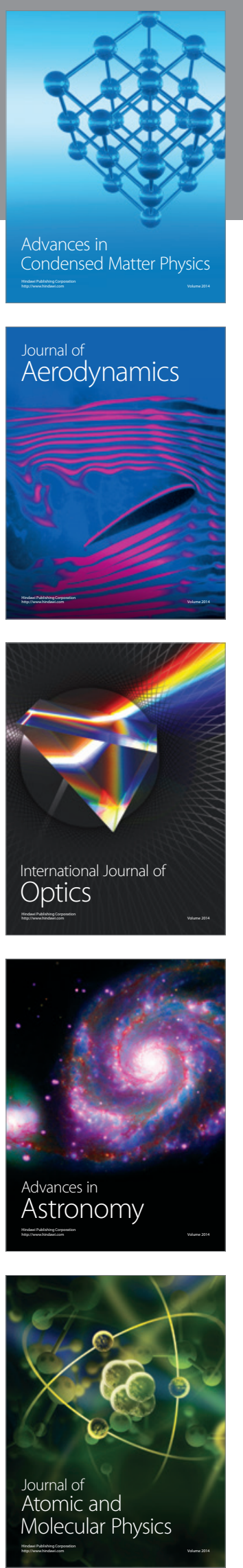